Claremont Colleges

Scholarship@ Claremont

All HMC Faculty Publications and Research

HMC Faculty Scholarship

$1-1-2007$

\title{
Exploring Physical Intuition in Elementary Pressure Vessels
}

Clive L. Dym

Harvey Mudd College

Harry E. Williams

Harvey Mudd College

\section{Recommended Citation}

Dym, Clive L. and Williams, Harry E., "Exploring Physical Intuition in Elementary Pressure Vessels" (2007). All HMC Faculty Publications and Research. Paper 965.

http://scholarship.claremont.edu/hmc_fac_pub/965

This Article - postprint is brought to you for free and open access by the HMC Faculty Scholarship at Scholarship @ Claremont. It has been accepted for inclusion in All HMC Faculty Publications and Research by an authorized administrator of Scholarship @ Claremont. For more information, please contact scholarship@cuc.claremont.edu. 


\title{
Exploring physical intuition in elementary pressure vessels
}

\author{
Clive L. Dym (corresponding author) and Harry E. Williams \\ Department of Engineering, Harvey Mudd College, Claremont, CA 91711, USA \\ E-mail: clive_dym@hmc.edu
}

\begin{abstract}
It is shown that displacement calculations for two classical 'chestnuts' - thick cylinders and spheres under internal and external pressures - present results that are not easily anticipated. Thus, such analyses provide an interesting opportunity for students (and teachers) taking elementary and advanced courses in the strength of materials to explore and perhaps enhance their physical intuition.
\end{abstract}

Keywords pressurized cylinders; pressurized spheres; physical intuition

\section{Introduction}

Courses in the strength of materials almost always include studies of the effects of internal and external pressure on circular cylinders and spheres. In elementary or first courses, the analyses are typically for very thin shell structures, and the modeling is aimed toward determining stress components. In particular, it is shown that the in-plane stresses (circumferential and axial for cylinders, circumferential and meridional for spheres) are larger than the transverse normal stress by a factor proportional to the radius-to-thickness $(R / h)$ ratio. In advanced courses in the strength of materials and applied elasticity, the stress distributions through thick cylinders and spheres are calculated, and the limiting thin-shell results are derived from them. The corresponding displacements (and strains) are rarely, if ever, discussed. Inasmuch as displacements of thick-walled cylinders are not usually a significant design issue, this is an understandable if regrettable omission.

However, it turns out that the consideration of displacements and the resulting geometry questions provide an interesting opportunity for students (and their teachers) to test and enhance their intuition. The basic questions are two:

- For an internally pressurized thick cylinder or sphere, does a point on the loaded (inner) surface move more than or less than a point on the unloaded (outer) surface?

- For an externally pressurized thick cylinder or sphere, does a point on the loaded (outer) surface move more than or less than a point on the unloaded (inner) surface?

The answers to these two questions are not as obvious as it first appears, and they differ with the load (i.e., internal versus external pressure) and vary with Poisson's ratio, $v$. For a shell made of an incompressible material (i.e., $v=0.5$ ), the overall shell volume must be preserved, which suggests that changes, $\Delta b$, of the outer radius, $b$, are accompanied by corresponding changes, $\Delta a$, of the inner radius, $a$ : 


$$
\begin{gathered}
(\Delta a)_{\text {cylinder }}=\left(\frac{b}{a}\right)(\Delta b)_{\text {cylinder }} \\
(\Delta a)_{\text {sphere }}=\left(\frac{b}{a}\right)^{2}(\Delta b)_{\text {sphere }}
\end{gathered}
$$

Since $b / a \geq 1$, equation 1 suggests that $\Delta b / \Delta a \leq 1$, independent of loading (i.e., whether the inner or outer surface is pressurized).

On the other hand, for $0 \leq v<0.5$, the stress-strain relations are coupled and local volume changes can occur. Thus, the in-plane strains depend on both in-plane and transverse normal stress components, which is reflected in changes in the effective stiffnesses of circular cylindrical arches and of spherical domes. Further, diffusion of the displacements from a loaded surface (sometimes called geometric spreading) suggests that the near displacement of a loaded surface should larger than the far displacement of the unloaded surface:

$$
\begin{aligned}
& p_{\mathrm{i}} \neq 0, p_{\mathrm{o}}=0: \frac{\Delta a}{\Delta b}>1 \\
& p_{\mathrm{i}}=0, p_{\mathrm{o}} \neq 0: \frac{\Delta b}{\Delta a}>1
\end{aligned}
$$

Clearly, the complete answers to the questions posed above are not easily and intuitively obvious, and further investigation is needed to clarify the issue.

\section{Pressurized circular cylinders}

Consider a hollow circular cylinder of inner radius $a$ and outer radius $b$, pressurized on its inner surface by a uniform pressure $p_{\mathrm{i}}$ and on its outer surface by a uniform pressure $p_{0}$. The radial and circumferential pressures for this axisymmetric problem are given in many places (e.g. [1-3]) as:

$$
\begin{gathered}
\sigma_{r r}=\frac{p_{\mathrm{i}} a^{2}}{b^{2}-a^{2}}\left(1-\frac{b^{2}}{r^{2}}\right)-\frac{p_{\mathrm{o}} b^{2}}{b^{2}-a^{2}}\left(1-\frac{a^{2}}{r^{2}}\right) \\
\sigma_{\theta \theta}=\frac{p_{\mathrm{i}} a^{2}}{b^{2}-a^{2}}\left(1+\frac{b^{2}}{r^{2}}\right)-\frac{p_{\mathrm{o}} b^{2}}{b^{2}-a^{2}}\left(1+\frac{a^{2}}{r^{2}}\right)
\end{gathered}
$$

If the cylinder is long and a state of plane strain is assumed along the axis, the radial displacement, $u_{\mathrm{c}}(r)$, can be determined from:

$$
u_{\mathrm{c}}(r)=r \varepsilon_{\theta \theta}=\frac{(1+v)\left((1-v) \sigma_{\theta \theta}-v \sigma_{r r}\right)}{E} r
$$

Then the radial displacements for the two separate cases of internal pressure (only) and external pressure (only) are found by substituting appropriate portions of equation 3 into equation 4 , yielding: 


$$
u_{\mathrm{ci}}(r)=\frac{(1+v) p_{\mathrm{i}} a^{2}}{E\left(b^{2}-a^{2}\right)}\left[(1-2 v) r+\frac{b^{2}}{r}\right]
$$

and

$$
u_{\mathrm{co}}(r)=-\frac{(1+v) p_{\mathrm{o}} b^{2}}{E\left(b^{2}-a^{2}\right)}\left[(1-2 v) r+\frac{a^{2}}{r}\right]
$$

Note the different signs of these results, which provides one opportunity to explore students' physical intuition.

Now, all that is needed to compare the radial displacements at the inner and outer surfaces is that equation $5 \mathrm{a}$ or equation $5 \mathrm{~b}$ be evaluated at $r=a$ and $r=b$, respectively, and that the appropriate ratio be constructed. For the case of internal pressure (only), that ratio is:

$$
\boldsymbol{R}_{\mathrm{ci}}=\frac{u_{\mathrm{ci}}(b)}{u_{\mathrm{ci}}(a)}=\left(\frac{\Delta(b)}{\Delta(a)}\right)_{\mathrm{ci}}=\frac{2(1-v) \rho}{(1-2 v)+\rho^{2}}
$$

where $\rho=b / a$, that is, the ratio of the cylinder's outer radius to its inner radius, which perforce is such that $\rho>1$. The relative size of the two displacements can be gauged according to whether the ratio $\boldsymbol{R}_{\mathrm{ci}}$ exceeds unity or not. The boundary for that measure is:

$$
\rho^{2}-2(1-v) \rho+(1-2 v)=0
$$

which has two roots:

$$
\rho_{1,2}=(1-2 v), 1
$$

The first root has no physical significance since the physics of the problem require that $\rho>1$. Further, since

$$
\left.\frac{\partial \boldsymbol{R}_{\mathrm{ci}}}{\partial \rho}\right|_{\rho=1}=-\frac{v}{(1-v)}<0
$$

it follows that the ratio $\boldsymbol{R}_{\mathrm{ci}}$ always decreases with $\rho$, and hence $u_{\mathrm{ci}}(b)<u_{\mathrm{ci}}(a)$, that is, the displacement at the outer radius is always smaller than that at the shell's inner, loaded radius.

For the case of external pressure (only), the ratio of inner and outer radial displacements is:

$$
\boldsymbol{R}_{\mathrm{co}}=\frac{u_{\mathrm{co}}(a)}{u_{\mathrm{co}}(b)}=\left(\frac{\Delta(a)}{\Delta(b)}\right)_{\mathrm{co}}=\frac{2(1-v) \rho}{1+(1-2 v) \rho^{2}}
$$

The assessment of the magnitude of the ratio $\boldsymbol{R}_{\text {co }}$ with $\rho$ is not quite as straightforward as in the previous case. The relative size of the two displacements can again be gauged according to how the ratio $\boldsymbol{R}_{\mathrm{co}}$ compares to unity. In this case the boundary for that measure is:

$$
(1-2 v) \rho^{2}-2(1-v) \rho+1=0
$$

which has two physically tenable roots: 


$$
\rho_{1,2}=1,1 /(1-2 v)
$$

Since

$$
\left.\frac{\partial \boldsymbol{R}_{\mathrm{co}}}{\partial \rho}\right|_{\rho=1}=\frac{v}{(1-v)}>0
$$

then $\boldsymbol{R}_{\mathrm{co}}>1$ for thinner cylinders, for which $1<\rho<1 /(1-2 v)$, and $\boldsymbol{R}_{\mathrm{co}}<1$ for thicker cylinders, for which $1 /(1-2 v)<\rho$. Further, $\boldsymbol{R}_{\text {co }}$ reaches a peak value of 1.107 , when $\rho=\rho_{\mathrm{cr}}=1 / \sqrt{(1-2 v)}=1.581$. Thus, the displacement at the inner radius is always greater than that at the shell's outer, loaded radius for relatively thin cylinders, but it is always smaller than that at the outer radius for thicker cylinders. Indeed, the analysis clearly shows that as the outer radius increases indefinitely, the displacement at the inner radius tends to zero. In other words, a pressure applied at an infinitely large radius does not affect the inner radius at all.

\section{Pressurized spheres}

Consider now a hollow sphere of inner radius $a$ and outer radius $b$, pressurized on its inner surface by a uniform pressure $p_{\mathrm{i}}$ and on its outer surface by a uniform pressure $p_{0}$. The radial and circumferential pressures for this spherically symmetric problem are [4]:

$$
\begin{aligned}
& \sigma_{r r}=\frac{p_{\mathrm{i}} a^{3}}{b^{3}-a^{3}}\left(1-\frac{b^{3}}{r^{3}}\right)-\frac{p_{\mathrm{o}} b^{3}}{b^{3}-a^{3}}\left(1-\frac{a^{3}}{r^{3}}\right) \\
& \sigma_{\theta \theta}=\sigma_{\phi \phi}=\frac{p_{\mathrm{i}} a^{3}}{2\left(b^{2}-a^{3}\right)}\left(2+\frac{b^{3}}{r^{3}}\right)-\frac{p_{\mathrm{o}} b^{3}}{2\left(b^{3}-a^{3}\right)}\left(2+\frac{a^{3}}{r^{3}}\right)
\end{aligned}
$$

The radial displacement, $u(r)$, is determined from:

$$
u(r)=r \varepsilon_{\theta \theta}=r \varepsilon_{\phi \phi}=\frac{(1-v) \sigma_{\theta \theta}-v \sigma_{r r}}{E} r
$$

The radial displacements for the two separate cases of internal pressure (only) and external pressure (only) are found by substituting appropriate portions of equation 10 into equation 11, yielding:

$$
u_{\mathrm{si}}(r)=\frac{p_{\mathrm{i}} a^{3}}{E\left(b^{3}-a^{3}\right)}\left[(1-2 v) r+\frac{(1+v)}{2} \frac{b^{3}}{r^{2}}\right]
$$

and

$$
u_{\mathrm{so}}(r)=-\frac{p_{\mathrm{o}} a^{3}}{E\left(b^{3}-a^{3}\right)}\left[(1-2 v) r+\frac{(1+v)}{2} \frac{a^{3}}{r^{2}}\right]
$$

Note here, too, the difference in sign between these two results, replicating the results for cylinders presented in equation 5 .

International Journal of Mechanical Engineering Education 35/2 
Once again, all that is needed to compare the radial displacements at the inner and outer surfaces is that equation $12 \mathrm{a}$ or equation $12 \mathrm{~b}$ be evaluated at $r=a$ and $r$ $=b$, respectively, and that the appropriate ratio be constructed. For the case of internal pressure (only) on the sphere that ratio is:

$$
\boldsymbol{R}_{\mathrm{si}}=\frac{u_{\mathrm{si}}(b)}{u_{\mathrm{csi}}(a)}=\left(\frac{\Delta(b)}{\Delta(a)}\right)_{\mathrm{si}}=\frac{3(1-v) \rho}{2(1-2 v)+(1+v) \rho^{3}}
$$

where $\rho=b / a$ is the ratio of the sphere's outer radius to its inner radius and, again, $\rho>1$. The relative size of the two displacements can once again be gauged according to whether the ratio $\boldsymbol{R}_{\mathrm{si}}$ exceeds unity or not. The boundary for that measure is:

$$
(1+v) \rho^{3}-3(1-v) \rho+2(1-2 v)=0
$$

which can be partially factored into the form:

$$
(\rho-1)\left[\rho^{2}+\rho-\frac{2(1-2 v)}{(1+v)}\right]=0
$$

Obviously, one of the roots of equation $14 \mathrm{~b}$ is $\rho=1$. The other two roots are real but have no physical significance because one is negative and the other is less than unity, which violates the requirement that $\rho>1$. Since

$$
\left.\frac{\partial \boldsymbol{R}_{\mathrm{si}}}{\partial \rho}\right|_{\rho=1}=-2 v /(1-v)<0
$$

it follows that the ratio $\boldsymbol{R}_{\mathrm{si}}$ always decreases from unity as $\rho$ increases, hence $u_{\mathrm{si}}(b)$ $<u_{\mathrm{si}}(a)$, that is, the displacement at the inner radius is always greater than that the shell's outer radius.

Thus, for the case of external pressure (only) on a sphere, the ratio of inner and outer radial displacements is:

$$
R_{\mathrm{so}}=\frac{u_{\mathrm{so}}(a)}{u_{\mathrm{so}}(b)}=\left(\frac{\Delta(a)}{\Delta(b)}\right)_{\mathrm{so}}=\frac{3(1-v) \rho^{2}}{(1+v)+2(1-2 v) \rho^{3}}
$$

The assessment of the magnitude of the ratio $\boldsymbol{R}_{\mathrm{so}}$ with $\rho$ is not quite as straightforward as in the previous case. The relative size of the two displacements can again be gauged according to how the ratio $\boldsymbol{R}_{\mathrm{so}}$ compares to unity. In this case, the boundary for that measure is:

$$
2(1-2 v) \rho^{3}-3(1-v) \rho^{2}+(1+v)=0
$$

which can also be partially factored as follows:

$$
(\rho-1)\left[\frac{2(1-2 v)}{(1+v)} \rho^{2}-\rho-1\right]=0
$$

International Journal of Mechanical Engineering Education 35/2 
As before, one of the roots of equation $16 \mathrm{~b}$ is $\rho=1$. Another of the roots is negative and thus of no interest. The third and final root is:

$$
\rho_{3}=\frac{(1+v)}{4(1-2 v)} 1+3 \sqrt{\frac{1-5 v / 3}{1+v}}
$$

For $1<\rho<\rho_{3}$, since

$$
\left.\frac{\partial \boldsymbol{R}_{\mathrm{so}}}{\partial \rho}\right|_{\rho=1}=\frac{2 v}{(1-v)}>0
$$

$\boldsymbol{R}_{\mathrm{so}}>1$, and the displacement at the inner radius is greater than that at the outer, loaded surface. $\boldsymbol{R}_{\mathrm{so}}$ reaches a peak value of 1.181 when $\rho=\rho_{\mathrm{cr}}=((1+v) /(1-2 v))^{1 / 3}$ $=1.481(v=0.30)$.

For $\rho>\rho_{3}, \boldsymbol{R}_{\mathrm{so}}<1$, and the displacement at the inner radius is smaller than that at the outer, loaded surface, and, as the outer radius increases indefinitely, the displacement at the inner radius tends to zero. In other words, a pressure applied at an infinitely large radius will not affect the inner radius at all.

\section{Conclusions}

The displacements for thick, elastic cylinders and spheres under internal and external pressures were calculated and presented. Analyses of the surface displacements demonstrated that predictions of their relative magnitudes are not easily made. Thus, such analyses may be useful for teachers of elementary and advanced courses in the strength of materials to help students test and improve their physical intuition.

\section{References}

[1] S. P. Timoshenko and J. N. Goodier, Theory of Elasticity (3rd edition) (McGraw-Hill, New York, 1934).

[2] J. M. Gere and S. P. Timoshenko, Mechanics of Materials (4th edition) (PWS Publishing Company, Boston, MA, 1997).

[3] R. D. Cook and W. C. Young, Advanced Mechanics of Materials (2nd edition) (Prentice Hall, Upper Saddle River, NJ, 1999).

[4] Y. C. Fung, Foundations of Solid Mechanics (Prentice-Hall, Englewood Cliffs, NJ, 1965). 